\title{
Nano-ZnO Catalyzed Multicomponent One-Pot Synthesis of Novel Spiro(indoline-pyranodioxine) Derivatives
}

\author{
Harshita Sachdeva, Rekha Saroj, and Diksha Dwivedi \\ Department of Chemistry, Faculty of Engineering and Technology, Mody Institute of Technology and Science, Lakshmangarh, Sikar, \\ Rajasthan 332311, India
}

Correspondence should be addressed to Harshita Sachdeva; drhmsachdevaster@gmail.com

Received 24 August 2013; Accepted 18 November 2013; Published 5 February 2014

Academic Editors: E. Gomez-Bengoa, G. B. Shul'pin, and A. Silva

Copyright (C) 2014 Harshita Sachdeva et al. This is an open access article distributed under the Creative Commons Attribution License, which permits unrestricted use, distribution, and reproduction in any medium, provided the original work is properly cited.

\begin{abstract}
A simple catalytic protocol for the synthesis of novel spiro[indoline-pyranodioxine] derivatives has been developed using $\mathrm{ZnO}$ nanoparticle as an efficient, green, and reusable catalyst. The derivatives are obtained in moderate to excellent yield by onepot three-component reaction of an isatin, malononitrile/ethylcyanoacetate, and 2,2-dimethyl-1,3-dioxane-4,6-dione in absolute ethanol under conventional heating and microwave irradiation. The catalyst was recovered by filtration from the reaction mixture and reused during five consecutive runs without any apparent loss of activity for the same reaction. The mild reaction conditions and recyclability of the catalyst make it environmentally benign synthetic procedure.
\end{abstract}

\section{Introduction}

Heterocyclic chemistry is one of the most complex and intriguing branches of organic chemistry and heterocyclic compounds constitute the largest and most varied family of organic compounds. Among heterocyclic compounds, indole derivatives exhibit a number of biological activities [1-8], for example, antimicrobial, anticonvulsant, antineoplastic, antiviral, antihypertensive, anti-inflammatory, and enzymatic inhibition activities dopaminergic agonist and so forth. In addition to substituted and condensed heterocycles, spiroindoles, with C-3 as spiro atom, have received considerable interest due to their strong biological activities [9-11].

Further, Meldrum's acid (2,2-dimethyl-1,3-dioxane-4,6diones) is useful in building block for peptide modification [12], synthesis of pseudopeptides [13], and antimicrobial and antitumoral natural products [14]. Similarly, alkylated Meldrum's acid has been encountered in the synthesis of dehydroar-juvabione [15], indane subunit containing paraquinonic acid ethyl ester, and deliquinone natural products [16]. Thus, 2,2-dimethyl-1,3-dioxane-4,6-diones efficiently serves in the synthesis of versatile intermediates and for the synthesis of pharmacologically active molecules.
The recent literature survey reveals that nano- $\mathrm{ZnO}$ as heterogeneous catalyst has received considerable attention because of its ecofriendly nature and has been explored as a powerful catalyst for several organic transformations [17-22]. To the best of our knowledge, there is no report available in the literature regarding the reaction of $1 \mathrm{H}$ indol-2,3-diones and activated methylene reagent (malononitrile/ethylcyanoacetate) with 2,2-dimethyl-1,3-dioxane-4,6diones.

Hence, prompted by these observations and in continuation to our interest in organic synthesis by different methods with the use of nanocatalyst [23], we report an easy and rapid catalytic application of $\mathrm{ZnO}$ nanoparticles for one-pot synthesis of spiroindole derivatives incorporating pyranodioxine by the reaction of $1 \mathrm{H}$-indole-2,3dione, malononitrile/ethylcyanoacetate, and Meldrum's acid in absolute ethanol under microwave irradiation and conventional heating (Scheme 1). The overall process involves the Knoevenagel condensation of Meldrum's acid with $1 H$ indole-2,3-dione followed by "in situ" Michael addition of malononitrile/ethyl cyanoacetate in single operation to give spiro(indole-pyranodioxine) derivatives similar to the earlier reports $[24,25]$ of the formation of spiroindoles in the 


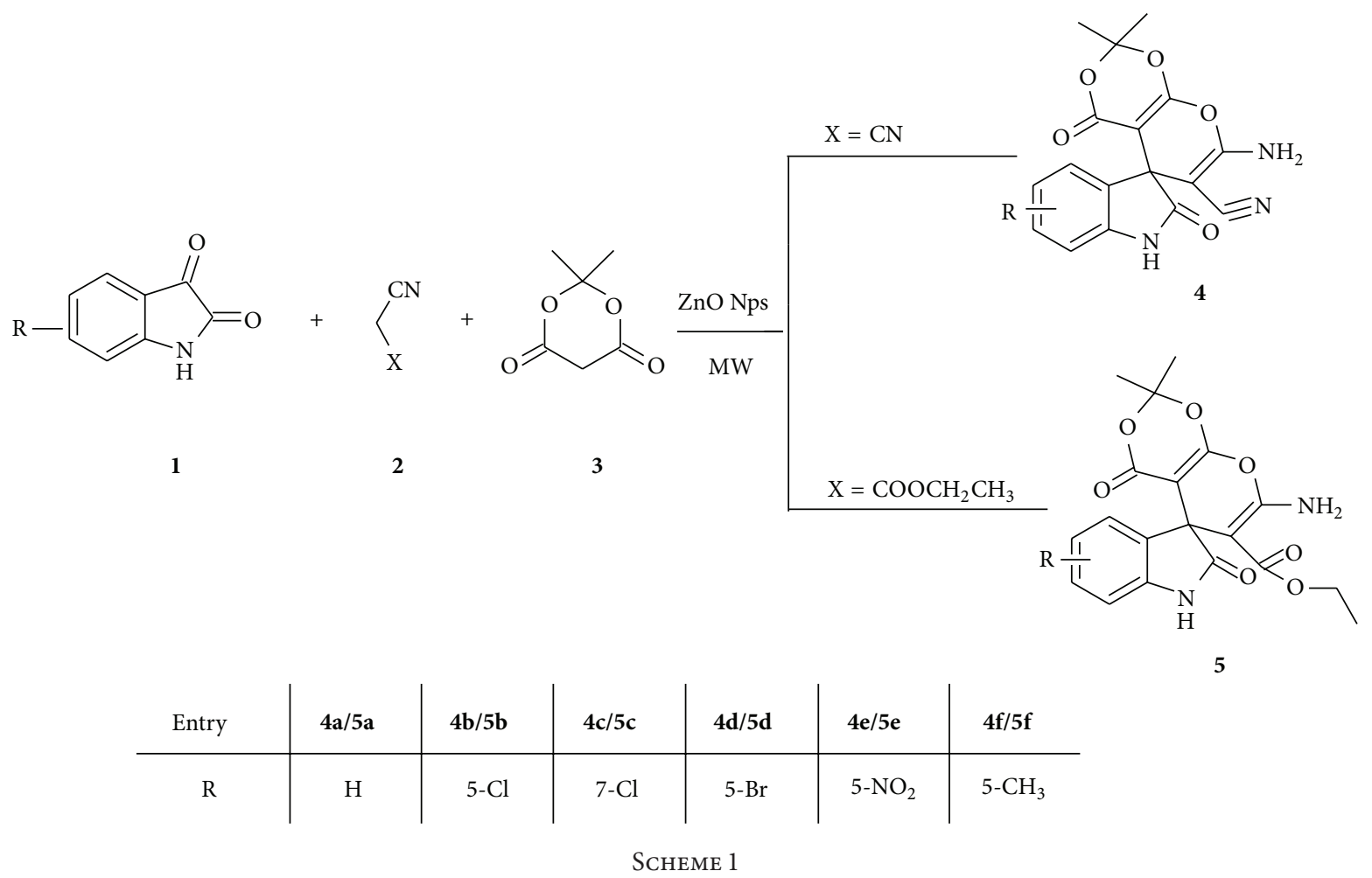

reaction of 3-carboethoxycyanomethylene- $2 \mathrm{H}$-indol-2-ones with cyclic ketones under classical conditions (Scheme 1). The process described here offers rapid facile one-pot synthesis of spiroindole derivatives using easily recyclable $\mathrm{ZnO}$ nanoparticles. This process is cost effective and hence ecofriendly as it is one-pot synthesis with easy workup and does not require harsh reagents. The process developed by us requires less quantity of catalyst $(30 \mathrm{mg}$ ) for carrying out the catalytic reaction, thus decreasing the amount of effluent to considerable level.

\section{Results and Discussion}

The reaction of $1 H$-indole-2,3-dione (1), malononitrile/ethylcyanoacetate (2), and 2,2-dimethyl-1,3-dioxane-4,6-diones (3) was examined in the presence of catalytic amount (30 mg) of $\mathrm{ZnO}$ nanoparticle under microwave irradiation and conventional heating to give novel $7^{\prime}$-amino- $2^{\prime} 2^{\prime}$-dimethyl$2,4^{\prime}$-dioxo-1,2-dihydrospiro[indoline- $3,5^{\prime}$-pyrano[2,3-d] $\left[1^{\prime}\right.$, $3^{\prime}$ ] dioxine] $-6^{\prime}$-carbonitrile/carboxyethylester (4a-f)/(5a-f) (Scheme 1) (Table 1).

To obtain the optimal conditions, the synthesis of $\mathbf{4 a}$ and 5a was used as a model reaction. A mixture of 1,2, and 3 in the presence of $\mathrm{ZnO}$ nanoparticles $(30 \mathrm{mg}$ ) was either refluxed for $10 \mathrm{hrs}$ or irradiated inside microwave oven for 9 min resulting in the formation of $4 \mathbf{a}$ in 62 or $87 \%$ yield, respectively (Table 2 ).

In order to confirm the effective involvement of $\mathrm{ZnO}$ nanoparticle during this transformation, a control experiment was conducted in the absence of $\mathrm{ZnO}$ nanoparticle for 4a, the reaction did not proceed, and the substrate remained unchanged even after 35 minutes of microwave irradiation and $25 \mathrm{hrs}$ of conventional heating (Table 2), while good results were obtained in the presence of $\mathrm{ZnO}$ nanoparticles. After some preliminary experiments, we found that a mixture of $1 H$-indole-2,3-dione, malononitrile, and 2,2-dimethyl-1,3dioxane-4,6-diones in the presence of $\mathrm{ZnO}$ nanoparticle afforded products in $87 \%$ yield under microwave irradiation (Table 1).

Encouraged by these results, we have extended this reaction to variously substituted $1 \mathrm{H}$-indole-2,3-diones under similar conditions to furnish the respective spiro(indolepyranodioxine) derivatives in excellent yields (81-88\%) using $\mathrm{ZnO}$ nanoparticle as a catalyst under microwave irradiation (Table 1).

Compounds were also synthesized under conventional heating using $\mathrm{ZnO}$ nanoparticle but yield of the product was found to be low (62-71\%) as compared to that obtained under microwave irradiation. The synthesis of compound $\mathbf{4 a}$ was carried out by refluxing for $10 \mathrm{hrs}$ resulting in $62 \%$ yield, while under microwave irradiation, reaction took 9 min with $87 \%$ yield of the product. It showed that microwave irradiation was found to have a beneficial effect on the synthesis of spiro(indole-pyranodioxine) derivatives (Table 2).

On optimizing the amount of catalyst, we found that $30 \mathrm{mg}$ of $\mathrm{ZnO}$ nanoparticles could effectively catalyze the reaction for the synthesis of desired product. With the inclusion of $10 \mathrm{mg}$ and $20 \mathrm{mg}$, reaction took longer time. Using more than $30 \mathrm{mg}$ has less effect on the yield and time of the reaction. Therefore, $30 \mathrm{mg}$ of $\mathrm{ZnO}$ nanoparticles was 
TABLE 1: Experimental data of $7^{\prime}$-amino- $2^{\prime} 2^{\prime}$-dimethyl-2,4' dioxo-1,2-dihydrospiro[indoline-3, $5^{\prime}$-pyrano[2,3-d]-1 $1^{\prime}, 3^{\prime}$ dioxine]-6 $6^{\prime}$-carbonitrile (4a-f)/carboxyethylester (5a-f) under microwave irradiation (Method A) and conventional heating (Method B).

\begin{tabular}{|c|c|c|c|c|c|c|}
\hline \multirow[b]{2}{*}{ Entry } & \multirow[b]{2}{*}{$\mathrm{X}$} & \multicolumn{2}{|c|}{ Method A (MW) } & \multicolumn{2}{|c|}{ Method B $(\Delta)$} & \multirow[b]{2}{*}{ M.P. $\left({ }^{\circ} \mathrm{C}\right)$} \\
\hline & & $\begin{array}{c}\text { Yield } \\
(\%)\end{array}$ & $\begin{array}{l}\text { Time } \\
\text { (min.) }\end{array}$ & $\begin{array}{c}\text { Yield } \\
(\%)\end{array}$ & $\begin{array}{l}\text { Time } \\
\text { (hrs.) }\end{array}$ & \\
\hline $4 a$ & & 87 & 9 & 62 & 10 & 294 \\
\hline
\end{tabular}

$4 b$<smiles>CC1(C)OC(=O)C2=C(OC(N)=C(C#N)C23C(=O)Nc2ccc(Cl)cc23)O1</smiles>

85<smiles>CC1(C)OC(=O)C2=C(OC(N)=C(C#N)C23C(=O)Nc2c(Cl)cccc23)O1</smiles>

83

3

86

10

65

$\sqrt{2}+x^{2}$

299

4d<smiles>CC1(C)OC(=O)C2=C(OC(N)=C(C#N)C23C(=O)Nc2ccc(Br)cc23)O1</smiles>

10

64

10

305

$4 e$<smiles>CC1(C)OC(=O)C2=C(OC(N)=C(C#N)C23C(=O)Nc2ccc([N+](=O)[O-])cc23)O1</smiles>

81

11

63

10

315

302 
TABle 1: Continued.

\begin{tabular}{|c|c|c|c|c|c|c|}
\hline \multirow[b]{2}{*}{ Entry } & \multirow[b]{2}{*}{ X } & \multicolumn{2}{|c|}{ Method A (MW) } & \multicolumn{2}{|c|}{ Method B $(\Delta)$} & \multirow[b]{2}{*}{ M.P. $\left({ }^{\circ} \mathrm{C}\right)$} \\
\hline & & $\begin{array}{c}\text { Yield } \\
(\%)\end{array}$ & $\begin{array}{l}\text { Time } \\
\text { (min.) }\end{array}$ & $\begin{array}{c}\text { Yield } \\
(\%)\end{array}$ & $\begin{array}{l}\text { Time } \\
\text { (hrs.) }\end{array}$ & \\
\hline $4 f$ & & 88 & 9 & 66 & 8 & 318 \\
\hline
\end{tabular}

$5 a$<smiles>CCOC(=O)C1=C(N)OC2=C(C(=O)OC(C)(C)O2)C12C(=O)Nc1ccccc12</smiles>

$5 b$<smiles>CCOC(=O)C1=C(N)OC2=C(C(=O)OC(C)(C)O2)C12C(=O)Nc1ccc(Cl)cc12</smiles>

$5 c$<smiles>CCOC(=O)C1=C(N)OC2=C(C(=O)OC(C)(C)O2)C12C(=O)Nc1c(Cl)cccc12</smiles>

$5 d$<smiles>CCOC(=O)C1=C(N)OC2=C(C(=O)OC(C)(C)O2)C12C(=O)Nc1ccc(Br)cc12</smiles>

$5 e$<smiles>CCOC(=O)C1=C(N)OC2=C(C(=O)OC(C)(C)O2)C12C(=O)Nc1ccc([N+](=O)[O-])cc12</smiles> 
TABle 1: Continued.

\begin{tabular}{|c|c|c|c|c|c|c|}
\hline \multirow[b]{2}{*}{ Entry } & \multirow[b]{2}{*}{$\mathrm{X}$} & \multicolumn{2}{|c|}{ Method A (MW) } & \multicolumn{2}{|c|}{ Method B $(\Delta)$} & \multirow[b]{2}{*}{ M.P. $\left({ }^{\circ} \mathrm{C}\right)$} \\
\hline & & $\begin{array}{c}\text { Yield } \\
(\%)\end{array}$ & $\begin{array}{l}\text { Time } \\
\text { (min.) }\end{array}$ & $\begin{array}{c}\text { Yield } \\
(\%) \\
\end{array}$ & $\begin{array}{l}\text { Time } \\
\text { (hrs.) }\end{array}$ & \\
\hline $5 f$ & & 86 & 10 & 71 & 9 & 319 \\
\hline
\end{tabular}

Reaction conditions: Meldrum's acid ( $1.0 \mathrm{mmol})$, isatin $(1.0 \mathrm{mmol})$, malononitrile/ethylcyanoacetate (1.0 mmol), absolute ethanol (15 mL), and catalyst (30 mg). ${ }^{a}$ Reaction under microwave irradiation was carried out at 420 watts.

TABLE 2: Comparison of catalytic activity of $\mathrm{ZnO}$ nanoparticles in the synthesis of compounds $\mathbf{4 a}$ and $\mathbf{5 a}$ by conventional heating $(\Delta)$ and microwave irradiation method (MW).

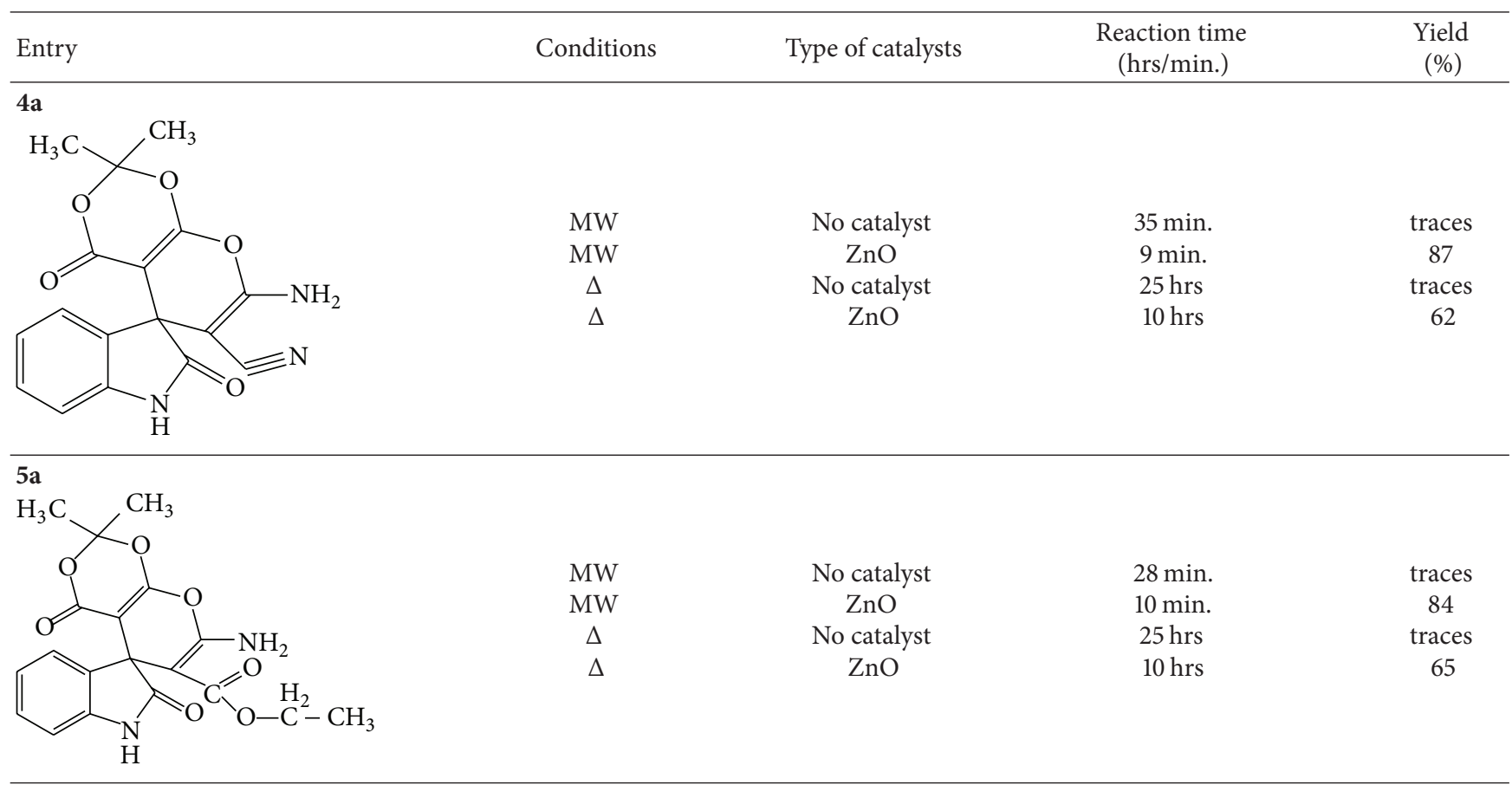

Amount of Reactants: Meldrum acid $(1.0 \mathrm{mmol})$, isatin $(1.0 \mathrm{mmol})$, and malononitrile/ethylcyanoacetate $(1.0 \mathrm{mmol})$; reaction under microwave irradiation was carried out at 420 watts.

sufficient to push the reaction forward, and further increasing of the amount of $\mathrm{ZnO}$ nanoparticles did not increase the yields (Table 3 ).

Reusability is one of the most important properties of this catalyst. To study the recyclability of the catalyst, the $\mathrm{ZnO}$ nanoparticles were used for the same reaction repeatedly and the change in their catalytic activity was studied. The relation between the number of cycles of the reaction and the catalytic activity in terms of yield of product is presented in Figure 1 . The catalyst recovered by filtration from the reaction mixture after dilution with ethyl acetate was reused as such for subsequent experiments under similar conditions. The catalyst retained optimum activity till five cycles after which drop in yield was observed (Figure 1).

A conceivable mechanism for the formation of the product would be as follows. The $\mathrm{ZnO}$ nanoparticle facilitate the Knoevenagel type coupling through Lewis acid sites $\left(\mathrm{Zn}^{+2}\right)$ coordinated to the oxygen of carbonyl groups. On the other hand, $\mathrm{ZnO}$ nanoparticles can activate methylene compounds so that deprotonation of the $\mathrm{C}-\mathrm{H}$ bond occurs in the presence of Lewis basic sites $\left(\mathrm{O}^{-2}\right)$. As a result, the formation of spiroindole derivatives proceeds by activation of reactants through both Lewis acids and basic sites of $\mathrm{ZnO}$ nanoparticles. 
TABLE 3: Optimization of the $\mathrm{ZnO}$ nanoparticle catalyzed model reaction for synthesis of $7^{\prime}$-amino- $2^{\prime} 2^{\prime}$-dimethyl-2, $4^{\prime}$-dioxo1,2-dihydrospiro[indoline-3, $5^{\prime}$ - pyrano [2,3-d] $\left[1^{\prime}, 3^{\prime}\right]$ dioxine] $-6^{\prime}$ carbonitrile (4a).

\begin{tabular}{lcc}
\hline Entry & Catalyst $(\mathrm{mg})$ & Yield (\%) \\
\hline 1 & No catalyst & - \\
2 & 10 & 80 \\
3 & 15 & 81 \\
4 & 20 & 83 \\
5 & 25 & 86 \\
6 & 30 & 89 \\
\hline
\end{tabular}

Amount of Reactants: Meldrum acid $(1.0 \mathrm{mmol})$, isatin $(1.0 \mathrm{mmol})$, malononitrile $(1.0 \mathrm{mmol})$, and absolute ethanol $(15 \mathrm{~mL})$.

\section{Experimental}

3.1. General. Reagents and solvents were obtained from commercial sources and used without further purification. Melting points were determined on a Toshniwal apparatus. The spectral analyses of synthesized compounds have been carried out at SAIF, Punjab University, Chandigarh. Purity of all compounds was checked by TLC using " $G$ " coated glass plates and benzene: ethyl acetate $(8: 2)$ as eluent. IR spectra were recorded in $\mathrm{KBr}$ on a Perkin Elmer Infrared RXI FTIR spectrophotometer (Figure 3 ) and ${ }^{1}$ HNMR spectra were recorded on Bruker Avance II 400 NMR spectrometer using DMSO- $\mathrm{d}_{6}$ and $\mathrm{CDCl}_{3}$ as solvent and tetramethylsilane (TMS) as internal reference standard. The obtained products were identified from their spectral $\left({ }^{1} \mathrm{HNMR},{ }^{13} \mathrm{C}\right.$ NMR, and IR) data. The microwave-assisted reactions were carried out in a Catalysts Systems Scientific Multimode MW oven attached with a magnetic stirrer and reflux condenser, operating at $700 \mathrm{~W}$ generating $2450 \mathrm{MHz}$ frequency.

3.2. General Procedure for the Synthesis of $\mathrm{ZnO}$ Nanoparticle. Nanoparticles were synthesized by the literature method [26]. Zinc acetate dihydrate, sodium hydroxide, CTAB, and the other reagents used were all analytical grade (from Shanghai Chemical Corp.) without further purification and reactions were carried out in air. In a typical synthesis, zinc acetate dihydrate, CTAB, and sodium hydroxide were mixed (molar ratio 1:0.4:3) and ground together in an agate mortar for $50 \mathrm{~min}$ at room temperature $\left(25^{\circ} \mathrm{C}\right)$. The reaction started readily during the mixing process, accompanied by the release of heat. The mixture was washed with distilled water in an ultrasonic bath. Finally, the product was dried in air at $60^{\circ} \mathrm{C}$ for $2 \mathrm{hrs}$.

Synthesis of $7^{\prime}$-Amino-2' $2^{\prime}$-dimethyl-2, $4^{\prime}$ dioxo-1,2-dihydrospiro[indoline-3, $5^{\prime}$-pyrano $[2,3-d]-\left[1^{\prime}, 3^{\prime}\right]$ dioxine]- $6^{\prime}$-carbonitrile/carboxyethylester $(\mathbf{4 a}-\boldsymbol{f}) /(\mathbf{5 a}-\boldsymbol{f})$ (see Scheme 2). Compounds $\mathbf{4 a - f}$ and $\mathbf{5 a}-\mathbf{f}$ were prepared by two different methods.

Method A: Microwave Irradiation Method. An equimolar mixture of $1 \mathrm{H}$-indole-2,3-dione (1) (1 mmole), malono- nitrile/ethylcynoacetate (1 mmole) (2), and 2,2-dimethyl-1,3dioxane-4,6-diones (1 mmole) (3) taken in absolute ethanol $(15 \mathrm{~mL})$ in presence of $\mathrm{ZnO}$ nanoparticle $(30 \mathrm{mg}$ ) was charged into a glass microwave vessel and refluxed inside a microwave oven at 420 watts for 9-10 min. Progress of the reaction was monitored by TLC. After completion of reaction, the reaction mixture was cooled to room temperature and solidified within an hour. The resulting solidified mixture was diluted with ethyl acetate $(5 \mathrm{~mL})$ and the catalyst was separated. The filtrate was evaporated on rotaevaporator to give a solid, which was dried and recrystallized from ethyl acetate.

Method B: Conventional Heating Method. An equimolar mixture of $1 \mathrm{H}$-indole-2,3-dione (1) (1 mmole), malononitrile/ethylcynoacetate (2) (1 mmole), and 2,2-dimethyl-1,3dioxane-4,6-diones (3) (1 mmole) taken in absolute ethanol $(15 \mathrm{~mL})$ in presence of $\mathrm{ZnO}$ nanoparticle $(30 \mathrm{mg})$ was refluxed for 10-11 hrs Progress of the reaction was monitored by TLC. After completion of reaction, the reaction mixture was cooled to room temperature and solidified within an hour. The resulting solidified mixture was diluted with ethyl acetate $(5 \mathrm{~mL})$ and the catalyst was separated. The filtrate was evaporated on rota-evaporator to give a solid, which was dried and recrystallized from ethyl acetate.

All the synthesized compounds were identified by their melting point, IR, ${ }^{1} \mathrm{HNMR},{ }^{13} \mathrm{CNMR}$, and mass spectral studies.

The spectroscopic characterization data of $(\mathbf{4 a - f} / 5 \mathbf{a}-\mathbf{f})$ are given below.

4 a $7^{\prime}$-amino-2' $2^{\prime}$-dimethyl-2, $4^{\prime}$-dioxo-1,2-dihydrospiro[indoline-3,5'-pyrano[2,3-d][1', $\left.3^{\prime}\right]$ dioxine $]-6^{\prime}$-carbonitrile. IR $\left(\mathrm{cm}^{-1}\right)$ : $3400\left(\mathrm{NH}_{2}\right), 3265(\mathrm{NH}), 3059$ (aromatic C-H str), 2984 (aliphatic C-H str), $2204(\mathrm{CN}), 1727$ (NH-C=O), 1620 $(\mathrm{O}=\mathrm{C}-\mathrm{O}), 1188.46(\mathrm{C}-\mathrm{O}-\mathrm{C}) ;{ }^{1} \operatorname{HNMR}(\delta \mathrm{ppm}): 10.93(\mathrm{~s}, 1 \mathrm{H}$, $\mathrm{NH}$ indole), 7.89 ( $\left.\mathrm{s}, 2 \mathrm{H}, \mathrm{NH}_{2}\right), 6.88-8.22(\mathrm{~m}, 4 \mathrm{H}, \mathrm{Ar}-\mathrm{H})$, $1.48\left(\mathrm{~s}, 3 \mathrm{H}, \mathrm{CH}_{3}\right), 1.90\left(\mathrm{~s}, 3 \mathrm{H}, \mathrm{CH}_{3}\right) ;{ }^{13} \mathrm{CNMR}(\delta \mathrm{ppm}): 166.4$ $(\mathrm{C}=\mathrm{O}), 159.4\left(\mathrm{C}-\mathrm{NH}_{2}\right), 124.0-141.6$ (aromatic carbons), 117.1 $(\mathrm{C} \equiv \mathrm{N}), 110.1(\mathrm{O}-\mathrm{C}-\mathrm{O}), 46.6$ (Spiro carbon), $26.3\left(\mathrm{CH}_{3}\right), 24.1$ $\left(\mathrm{CH}_{3}\right)$; Anal. Calcd. for $\mathrm{C}_{17} \mathrm{H}_{13} \mathrm{~N}_{3} \mathrm{O}_{5}$ : C, 60.1, H, 3.8, N, 12.3. Found: C, 60.01, H, 3.85, N, 12.37; MS: $[\mathrm{M}]^{+}$at $m / z 339$.

$4 \boldsymbol{b} 7^{\prime}$-Amino-2' $2^{\prime}$-dimethyl-2, $4^{\prime}$-dioxo-5-chloro-1,2-dihydrospiro[indoline-3, $5^{\prime}$-pyrano $[2,3-d]\left[1^{\prime}, 3^{\prime}\right]$ dioxine]- $6^{\prime}$-carbonitrile. IR $\left(\mathrm{cm}^{-1}\right)$ : $3410\left(\mathrm{NH}_{2}\right), 3260(\mathrm{NH}), 3053$ (aromatic C-H str), 2980 (aliphatic C-H str), $2202(\mathrm{CN}), 1720(\mathrm{NH}-\mathrm{C}=\mathrm{O})$, $1622(\mathrm{O}=\mathrm{C}-\mathrm{O}), 1180(\mathrm{C}-\mathrm{O}-\mathrm{C}) ;{ }^{1} \operatorname{HNMR}(\delta \mathrm{ppm}): 10.84(\mathrm{~s}$, $1 \mathrm{H}, \mathrm{NH}$ indole), 7.78 (s, 2H, $\left.\mathrm{NH}_{2}\right), 6.82-8.20$ (m, 3H, Ar-H), $1.42\left(\mathrm{~s}, 3 \mathrm{H}, \mathrm{CH}_{3}\right), 1.94\left(\mathrm{~s}, 3 \mathrm{H}, \mathrm{CH}_{3}\right) ;{ }^{13} \mathrm{CNMR}(\delta \mathrm{ppm}): 166.4$ $(\mathrm{C}=\mathrm{O}), 159.4\left(\mathrm{C}-\mathrm{NH}_{2}\right), 124.0-141.6$ (aromatic carbons), 117.1 $(\mathrm{C} \equiv \mathrm{N}), 110.1(\mathrm{O}-\mathrm{C}-\mathrm{O}), 46.6$ (Spiro carbon), $26.3\left(\mathrm{CH}_{3}\right), 24.1$ $\left(\mathrm{CH}_{3}\right)$; Anal. Calcd. for $\mathrm{C}_{17} \mathrm{H}_{12} \mathrm{ClN}_{3} \mathrm{O}_{5}$ : C, 54.63, H, 3.24, N, 11.24. Found: C, 54.80, H, 3.26, N, 11.26; MS: $[\mathrm{M}]^{+}$at $\mathrm{m} / z$ 373.74 .

4c $7^{\prime}$-Amino-2' $2^{\prime}$-dimethyl-2, $4^{\prime}$-dioxo-7-chloro-1,2-dihydrospiro [indoline- $3,5^{\prime}$ - pyrano $[2,3-d]\left[1^{\prime}, 3^{\prime}\right]$ dioxine $]-6^{\prime}$-carboni- 


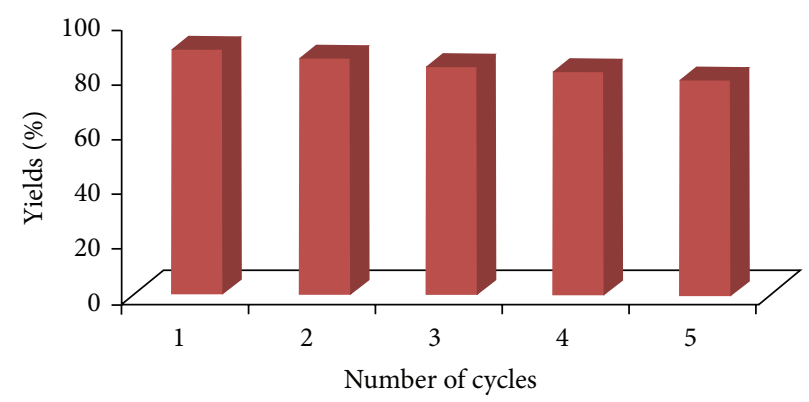

Figure 1: Recyclability of $\mathrm{ZnO}$ nanoparticles for the synthesis of $7^{\prime}$-amino- $2^{\prime} 2^{\prime}$-dimethyl-2, $4^{\prime}$ dioxo-1,2-dihydrospiro[indoline-3,5' pyrano[2,3-d] $\left[1^{\prime}, 3^{\prime}\right]$ dioxine $]-6^{\prime}$-carbonitrile (4a).

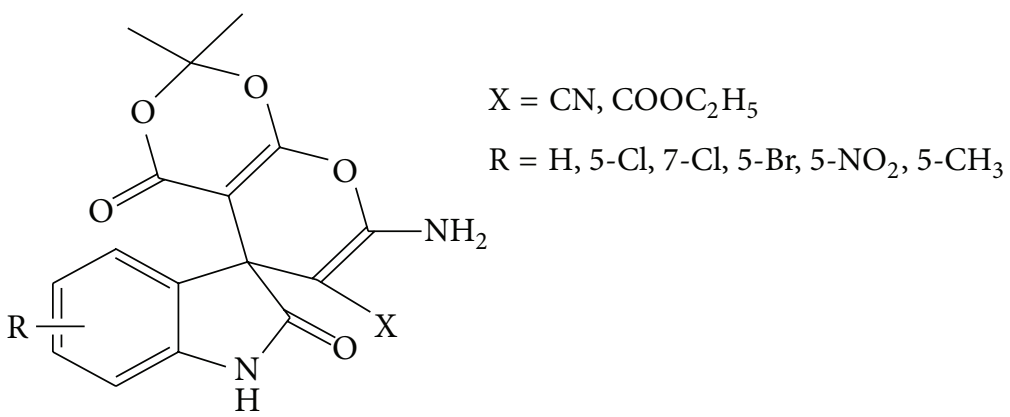

SCHEMe 2

trile. IR $\left(\mathrm{cm}^{-1}\right): 3402\left(\mathrm{NH}_{2}\right), 3256(\mathrm{NH}), 3039$ (aromatic $\mathrm{C}-\mathrm{H}$ str), 2977 (aliphatic C-H str), $2210(\mathrm{CN}), 1732(\mathrm{NH}-\mathrm{C}=\mathrm{O})$, $1610(\mathrm{O}=\mathrm{C}-\mathrm{O}), 1175(\mathrm{C}-\mathrm{O}-\mathrm{C}) ;{ }^{1} \mathrm{HNMR}(\delta \mathrm{ppm}): 10.78(\mathrm{~s}$, $1 \mathrm{H}, \mathrm{NH}$ indole), 7.76 (s, 2H, $\left.\mathrm{NH}_{2}\right), 6.84-8.26$ (m, 3H, Ar-H), $1.46\left(\mathrm{~s}, 3 \mathrm{H}, \mathrm{CH}_{3}\right), 1.92\left(\mathrm{~s}, 3 \mathrm{H}, \mathrm{CH}_{3}\right) ;{ }^{13} \mathrm{CNMR}(\delta \mathrm{ppm}): 166.4$ $(\mathrm{C}=\mathrm{O}), 159.4\left(\mathrm{C}-\mathrm{NH}_{2}\right), 124.0-141.6$ (aromatic carbons), 117.1 $(\mathrm{C} \equiv \mathrm{N}), 110.1$ (O-C-O), 46.6 (Spiro carbon), $26.3\left(\mathrm{CH}_{3}\right), 24.1$ $\left(\mathrm{CH}_{3}\right)$; Anal. Calcd. for $\mathrm{C}_{17} \mathrm{H}_{12} \mathrm{ClN}_{3} \mathrm{O}_{5}: \mathrm{C}, 54.63, \mathrm{H}, 3.24$, $\mathrm{N}, 11.24$. Found: C, $54.79, \mathrm{H}, 3.27, \mathrm{~N}, 11.26$; MS: $[\mathrm{M}]^{+}$at $\mathrm{m} / z$ 373.74 .

$4 d 7^{\prime}$-Amino-2 $2^{\prime}$-dimethyl-2, $4^{\prime}$-dioxo-5-bromo-1,2-dihydrospiro [indoline- $3,5^{\prime}$ - pyrano $[2,3-d]\left[1^{\prime}, 3^{\prime}\right]$ dioxine $]-6^{\prime}$-carbonitrile. IR $\left(\mathrm{cm}^{-1}\right)$ : $3400\left(\mathrm{NH}_{2}\right), 3280(\mathrm{NH}), 3050$ (aromatic C-H str), 2974 (aliphatic C-H str), 2200 (CN), 1722 (NH$\mathrm{C}=\mathrm{O}), 1618$ (O=C-O), $1180(\mathrm{C}-\mathrm{O}-\mathrm{C}) ;{ }^{1} \mathrm{HNMR}(\delta \mathrm{ppm})$ : $10.74\left(\mathrm{~s}, 1 \mathrm{H}, \mathrm{NH}\right.$ indole), $7.72\left(\mathrm{~s}, 2 \mathrm{H}, \mathrm{NH}_{2}\right), 6.87-8.26(\mathrm{~m}$, $3 \mathrm{H}, \mathrm{Ar}-\mathrm{H}), 1.40\left(\mathrm{~s}, 3 \mathrm{H}, \mathrm{CH}_{3}\right), 1.98\left(\mathrm{~s}, 3 \mathrm{H}, \mathrm{CH}_{3}\right) ;{ }^{13} \mathrm{CNMR}$ $\left(\delta\right.$ ppm): $166.4(\mathrm{C}=\mathrm{O}), 159.4\left(\mathrm{C}-\mathrm{NH}_{2}\right), 124.0-141.6$ (aromatic carbons), $117.1(\mathrm{C} \equiv \mathrm{N}), 110.1(\mathrm{O}-\mathrm{C}-\mathrm{O}), 46.6$ (Spiro carbon), $26.3\left(\mathrm{CH}_{3}\right), 24.1\left(\mathrm{CH}_{3}\right)$; Anal. Calcd. for $\mathrm{C}_{17} \mathrm{H}_{12} \mathrm{BrN}_{3} \mathrm{O}_{5}$ : C, 48.82, H, 2.89, N, 10.05. Found: C, 48.65, H, 2.91, N, 10.07; MS: $[\mathrm{M}]^{+}$at $m / z 418$.

4 e $7^{\prime}$-Amino-2' $2^{\prime}$-dimethyl-2, $4^{\prime}$-dioxo-5-nitro-1,2-dihydrospiro[indoline-3, $5^{\prime}$-pyrano $[2,3-d]\left[1^{\prime}, 3^{\prime}\right]$ dioxine]- $6^{\prime}$-carbonitrile. IR $\left(\mathrm{cm}^{-1}\right)$ : $3400\left(\mathrm{NH}_{2}\right), 3265(\mathrm{NH}), 3059$ (aromatic C-H str), 2984 (aliphatic C-H str), 2204 (CN), 1727 (NH-C=O),
$1620(\mathrm{O}=\mathrm{C}-\mathrm{O}), 1188.46(\mathrm{C}-\mathrm{O}-\mathrm{C}) ;{ }^{1} \mathrm{HNMR}(\delta \mathrm{ppm}): 10.78(\mathrm{~s}$, $1 \mathrm{H}, \mathrm{NH}$ indole), 7.71 (s, 2H, $\left.\mathrm{NH}_{2}\right), 6.83-8.28(\mathrm{~m}, 3 \mathrm{H}, \mathrm{Ar}-\mathrm{H})$, $1.48\left(\mathrm{~s}, 3 \mathrm{H}, \mathrm{CH}_{3}\right), 1.90\left(\mathrm{~s}, 3 \mathrm{H}, \mathrm{CH}_{3}\right) ;{ }^{13} \mathrm{CNMR}(\delta \mathrm{ppm}): 166.4$ $(\mathrm{C}=\mathrm{O}), 159.4\left(\mathrm{C}-\mathrm{NH}_{2}\right), 124.0-141.6$ (aromatic carbons), 117.1 $(\mathrm{C} \equiv \mathrm{N}), 110.1$ (O-C-O), 46.6 (Spiro carbon), $26.3\left(\mathrm{CH}_{3}\right), 24.1$ $\left(\mathrm{CH}_{3}\right)$; Anal. Calcd. for $\mathrm{C}_{17} \mathrm{H}_{12} \mathrm{~N}_{4} \mathrm{O}_{7}: \mathrm{C}, 53.13, \mathrm{H}, 3.15, \mathrm{~N}$, 14.58. Found: C, 53.30, H, 3.17, N, 14.59; MS: [M] $]^{+}$at $m / z 384$.

If $7^{\prime}$-Amino-2' $2^{\prime}$-dimethyl-2, $4^{\prime}$-dioxo-5-methyl-1,2-dihydrospiro[indoline-3, $5^{\prime}$-pyrano $[2,3-d]\left[1^{\prime}, 3^{\prime}\right]$ dioxine]- $6^{\prime}$-carbonitrile. IR $\left(\mathrm{cm}^{-1}\right)$ : $3405\left(\mathrm{NH}_{2}\right), 3261(\mathrm{NH}), 3048$ (aromatic $\mathrm{C}-\mathrm{H}$ str), 2981 (aliphatic C-H str), $2206(\mathrm{CN}), 1724(\mathrm{NH}-\mathrm{C}=\mathrm{O})$, 1618 (O=C-O), 1178 (C-O-C); ${ }^{1} \mathrm{HNMR}(\delta \mathrm{ppm}): 10.81(\mathrm{~s}$, $1 \mathrm{H}, \mathrm{NH}$ indole), 7.75 (s, 2H, $\left.\mathrm{NH}_{2}\right), 6.81-8.20(\mathrm{~m}, 3 \mathrm{H}, \mathrm{Ar}-\mathrm{H})$, $2.35\left(\mathrm{~s}, 3 \mathrm{H}, \mathrm{CH}_{3}\right) 1.40\left(\mathrm{~s}, 3 \mathrm{H}, \mathrm{CH}_{3}\right), 1.98\left(\mathrm{~s}, 3 \mathrm{H}, \mathrm{CH}_{3}\right)$; ${ }^{13} \mathrm{CNMR}(\delta \mathrm{ppm}): 166.4(\mathrm{C}=\mathrm{O}), 159.4\left(\mathrm{C}-\mathrm{NH}_{2}\right), 124.0-141.6$ (aromatic carbons), $117.1(\mathrm{C} \equiv \mathrm{N}), 110.1(\mathrm{O}-\mathrm{C}-\mathrm{O}), 46.6$ (Spiro carbon), $26.3\left(\mathrm{CH}_{3}\right), 24.1\left(\mathrm{CH}_{3}\right), 25.1\left(\mathrm{CH}_{3}\right)$; Anal. Calcd. for $\mathrm{C}_{18} \mathrm{H}_{15} \mathrm{~N}_{3} \mathrm{O}_{5}$ : C, 61.19, H, 4.28, N, 11.89. Found: C, 61.40, H, 4.30, N, 11.87; MS: $[\mathrm{M}]^{+}$at $m / z 353$.

5 a $7^{\prime}$-Amino-2 $2^{\prime} 2^{\prime}$-dimethyl-2, $4^{\prime}$-dioxo-1,2-dihydrospiro[indoline-3,5'-pyrano[2,3-d][1',3'] dioxine $]-6^{\prime}$-carboxyethylester. IR $\left(\mathrm{cm}^{-1}\right)$ : $3402\left(\mathrm{NH}_{2}\right), 3298(\mathrm{NH}), 3059$ (aromatic C-H str), 2984 (aliphatic C-H str), 1727 ( $\mathrm{NH}-\mathrm{C}=\mathrm{O}), 1620$ (O=C-O), 1184 (C-O-C); ${ }^{1} \mathrm{HNMR}(\delta) 10.97$ (s, 1H, NH indole), 8.15 (s, $\left.2 \mathrm{H}, \mathrm{NH}_{2}\right), 6.92-7.46(\mathrm{~m}, 4 \mathrm{H}, \mathrm{Ar}-\mathrm{H}), 4.44\left(\mathrm{q}, 2 \mathrm{H}, \mathrm{CH}_{2}\right), 1.35$ $\left(\mathrm{t}, 3 \mathrm{H}, \mathrm{CH}_{3}\right), 1.18\left(\mathrm{~s}, 3 \mathrm{H}, \mathrm{CH}_{3}\right), 1.14\left(\mathrm{~s}, 3 \mathrm{H}, \mathrm{CH}_{3}\right) ;{ }^{13} \mathrm{CNMR}$ 


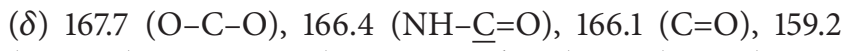
$\left(\mathrm{C}-\mathrm{NH}_{2}\right), 124.3-142.0$ (aromatic carbons), $61.2\left(\mathrm{OCH}_{2}\right), 46.6$ (spiro carbon), $26.3\left(\mathrm{CH}_{3}\right), 24.1\left(\mathrm{CH}_{3}\right), 14.1\left(\mathrm{CH}_{3}\right.$ ester); Anal. Calcd. for $\mathrm{C}_{19} \mathrm{H}_{18} \mathrm{~N}_{2} \mathrm{O}_{7}$ : C, 59.07, H, 4.70, N, 7.25. Found: C, 59.25, H, 4.68, N, 7.26; MS: $[\mathrm{M}]^{+}$at $m / z 386$.

$5 \boldsymbol{b} 7^{\prime}$-Amino-2' $2^{\prime}$-dimethyl-2, $4^{\prime}$-dioxo-5-chloro-1,2-dihydrospiro[indoline-3,5'-pyrano [2,3-d][1', $\left.3^{\prime}\right]$ dioxine]-6'-carboxyethylester. IR $\left(\mathrm{cm}^{-1}\right): 3400\left(\mathrm{NH}_{2}\right), 3292(\mathrm{NH}), 3050$ (aromatic C-H str), 2980 (aliphatic C-H str), $1722(\mathrm{NH}-\mathrm{C}=\mathrm{O}), 1622$ $(\mathrm{O}=\mathrm{C}-\mathrm{O}), 1182(\mathrm{C}-\mathrm{O}-\mathrm{C}) ;{ }^{1} \mathrm{HNMR}(\delta): 10.90$ (s, 1H, NH indole), 8.12 (s, $\left.2 \mathrm{H}, \mathrm{NH}_{2}\right), 6.92-7.26(\mathrm{~m}, 3 \mathrm{H}, \mathrm{Ar}-\mathrm{H}), 4.40$ (q, $\left.2 \mathrm{H}, \mathrm{CH}_{2}\right), 1.32\left(\mathrm{t}, 3 \mathrm{H}, \mathrm{CH}_{3}\right), 1.12\left(\mathrm{~s}, 3 \mathrm{H}, \mathrm{CH}_{3}\right), 1.10(\mathrm{~s}, 3 \mathrm{H}$, $\left.\mathrm{CH}_{3}\right) ;{ }^{13} \mathrm{CNMR}(\delta): 167.7(\mathrm{O}-\mathrm{C}-\mathrm{O}), 166.4(\mathrm{NH}-\mathrm{C}=\mathrm{O}), 166.1$ $(\mathrm{C}=\mathrm{O}), 159.2\left(\mathrm{C}-\mathrm{NH}_{2}\right), 124.3-142.0$ (aromatic carbons), 61.2 $\left(\mathrm{OCH}_{2}\right), 46.6$ (spiro carbon), $26.3\left(\mathrm{CH}_{3}\right), 24.1\left(\mathrm{CH}_{3}\right), 14.1$ $\left(\mathrm{CH}_{3}\right.$ ester); Anal. Calcd. for $\mathrm{C}_{19} \mathrm{H}_{17} \mathrm{ClN}_{2} \mathrm{O}_{7}$ : C, 54.23, $\mathrm{H}$, 4.07, N, 6.66. Found: C, 54.40, H, 4.05, N, 6.65; MS: $[\mathrm{M}]^{+}$at $\mathrm{m} / z 420.80$.

$5 c 7^{\prime}$-Amino-2 $2^{\prime}$-dimethyl-2, $4^{\prime}$-dioxo-7-chloro-1,2-dihydrospiro [indoline-3, $5^{\prime}$-pyrano $[2,3-d]\left[1^{\prime}, 3^{\prime}\right]$ dioxine]- $6^{\prime}$-carboxyethylester. IR $\left(\mathrm{cm}^{-1}\right): 3408\left(\mathrm{NH}_{2}\right), 3290(\mathrm{NH}), 3058$ (aromatic C-H str), 2984 (aliphatic C-H str), $1728(\mathrm{NH}-\mathrm{C}=\mathrm{O}), 1624$ $(\mathrm{O}=\mathrm{C}-\mathrm{O}), 1188(\mathrm{C}-\mathrm{O}-\mathrm{C}) ;{ }^{1} \mathrm{HNMR}(\delta): 10.92(\mathrm{~s}, 1 \mathrm{H}, \mathrm{NH}$ indole), 8.10 (s, $\left.2 \mathrm{H}, \mathrm{NH}_{2}\right), 6.90-7.20(\mathrm{~m}, 3 \mathrm{H}, \mathrm{Ar}-\mathrm{H}), 4.42$ (q, $\left.2 \mathrm{H}, \mathrm{CH}_{2}\right), 1.30\left(\mathrm{t}, 3 \mathrm{H}, \mathrm{CH}_{3}\right), 1.16\left(\mathrm{~s}, 3 \mathrm{H}, \mathrm{CH}_{3}\right), 1.10(\mathrm{~s}, 3 \mathrm{H}$, $\left.\mathrm{CH}_{3}\right) ;{ }^{13} \mathrm{CNMR}(\delta): 167.7(\mathrm{O}-\mathrm{C}-\mathrm{O}), 166.4(\mathrm{NH}-\mathrm{C}=\mathrm{O}), 166.1$ $(\mathrm{C}=\mathrm{O}), 159.2\left(\mathrm{C}-\mathrm{NH}_{2}\right), 124.3-140.0$ (aromatic carbons), 61.2 $\left(\mathrm{OCH}_{2}\right), 46.6$ (Spiro carbon), $26.3\left(\mathrm{CH}_{3}\right), 24.1\left(\mathrm{CH}_{3}\right), 14.1$ $\left(\mathrm{CH}_{3}\right.$ ester); Anal. Calcd. for $\mathrm{C}_{19} \mathrm{H}_{17} \mathrm{ClN}_{2} \mathrm{O}_{7}$ : C, 54.23, $\mathrm{H}$, 4.07, N, 6.66. Found: C, 54.42, H, 4.06, N, 6.64; MS: $[\mathrm{M}]^{+}$at $m / z 420.80$.

5d $7^{\prime}$-Amino-2' $2^{\prime}$-dimethyl-2, $4^{\prime}$-dioxo-5-bromo-1,2-dihydrospiro [indoline- $3,5^{\prime}$-pyrano $[2,3-d]\left[1^{\prime}, 3^{\prime}\right]$ dioxine $]-6^{\prime}$-carboxyethylester. IR $\left(\mathrm{cm}^{-1}\right): 3400\left(\mathrm{NH}_{2}\right), 3260(\mathrm{NH}), 3050$ (aromatic $\mathrm{C}-\mathrm{H}$ str), 2982 (aliphatic $\mathrm{C}-\mathrm{H}$ str), $1790(\mathrm{C}=\mathrm{O}), 1722(\mathrm{NH}-$ $\mathrm{C}=\mathrm{O}), 1618(\mathrm{O}=\mathrm{C}-\mathrm{O}), 1130(\mathrm{C}-\mathrm{O}-\mathrm{C}) ;{ }^{1} \operatorname{HNMR}(\delta): 10.74(\mathrm{~s}$, $1 \mathrm{H}, \mathrm{N}$ indole), $6.90\left(\mathrm{~s}, 2 \mathrm{H}, \mathrm{NH}_{2}\right), 6.87-8.26(\mathrm{~m}, 3 \mathrm{H}, \mathrm{Ar}-\mathrm{H})$, $4.19\left(\mathrm{q}, 2 \mathrm{H}, \mathrm{CH}_{2}\right), 1.98\left(\mathrm{~s}, 3 \mathrm{H}, \mathrm{CH}_{3}\right), 1.40\left(\mathrm{~s}, 3 \mathrm{H}, \mathrm{CH}_{3}\right), 1.34(\mathrm{t}$, $\left.3 \mathrm{H}, \mathrm{CH}_{3}\right) ;{ }^{13} \mathrm{CNMR}(\delta): 167.7(\mathrm{O}-\mathrm{C}-\mathrm{O}), 166.4(\mathrm{NH}-\mathrm{C}=\mathrm{O})$, $166.1(\mathrm{C}=\mathrm{O}), 159.2\left(\mathrm{C}-\mathrm{NH}_{2}\right), 124.3-142.0$ (aromatic carbons), $61.2\left(\mathrm{OCH}_{2}\right), 46.6$ (Spiro carbon), $26.3\left(\mathrm{CH}_{3}\right), 24.1\left(\mathrm{CH}_{3}\right)$, $14.1\left(\mathrm{CH}_{3}\right.$ ester); Anal. Calcd. for $\mathrm{C}_{19} \mathrm{H}_{17} \mathrm{BrN}_{2} \mathrm{O}_{7}$ : C, 49.05, H, 3.68, N, 6.02. Found: C, 49.24, H, 3.70, N, 6.04; MS: $[M]^{+}$ at $m / z 465$.

5e $7^{\prime}$-Amino-2' $2^{\prime}$-dimethyl-2, $4^{\prime}$-dioxo-5-nitro-1,2-dihydrospiro[indoline-3, $5^{\prime}$-pyrano $[2,3-d]\left[1^{\prime}, 3^{\prime}\right]$ dioxine]- $6^{\prime}$-carboxyethylester. IR $\left(\mathrm{cm}^{-1}\right): 3420\left(\mathrm{NH}_{2}\right), 3290(\mathrm{NH}), 3069$ (aromatic $\mathrm{C}-\mathrm{H}$ str), $1732(\mathrm{NH}-\mathrm{C}=\mathrm{O}), 1620(\mathrm{O}=\mathrm{C}-\mathrm{O}), 1180(\mathrm{C}-\mathrm{O}-\mathrm{C})$; ${ }^{1} \mathrm{HNMR}(\delta): 10.92$ (s, $1 \mathrm{H}, \mathrm{NH}$ indole), $8.14\left(\mathrm{~s}, 2 \mathrm{H}, \mathrm{NH}_{2}\right), 7.2-$ $8.08(\mathrm{~m}, 3 \mathrm{H}, \mathrm{Ar}-\mathrm{H}), 4.40\left(\mathrm{q}, 2 \mathrm{H}, \mathrm{CH}_{2}\right), 2.78(\mathrm{~s}, 1 \mathrm{H}, \mathrm{NH}), 1.38$ $\left(\mathrm{t}, 3 \mathrm{H}, \mathrm{CH}_{3}\right), 1.16\left(\mathrm{~s}, 3 \mathrm{H}, \mathrm{CH}_{3}\right), 1.10\left(\mathrm{~s}, 3 \mathrm{H}, \mathrm{CH}_{3}\right) ;{ }^{13} \mathrm{CNMR}$ $(\delta): 167.7(\mathrm{O}-\mathrm{C}-\mathrm{O}), 166.4(\mathrm{NH}-\mathrm{C}=\mathrm{O}), 166.1(\mathrm{C}=\mathrm{O}), 159.2$ $\left(\mathrm{C}-\mathrm{NH}_{2}\right), 124.3-142.0$ (aromatic carbons), $61.2\left(\mathrm{OCH}_{2}\right), 46.6$

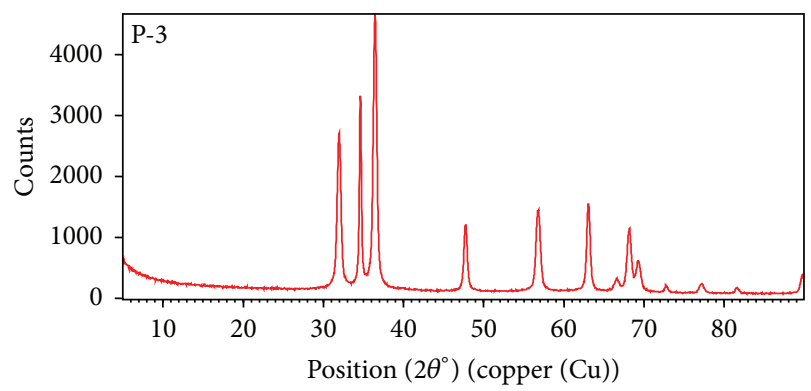

FIgURE 2: XRD pattern of $\mathrm{ZnO}$ nanoparticles.

(Spiro carbon), $26.3\left(\mathrm{CH}_{3}\right), 24.1\left(\mathrm{CH}_{3}\right), 14.1\left(\mathrm{CH}_{3}\right.$ ester); Anal. Calcd. for $\mathrm{C}_{19} \mathrm{H}_{17} \mathrm{~N}_{3} \mathrm{O}_{9}$ : C, 52.90, H, 3.97, N, 9.74. Found: C, 52.71, H, 3.95, N, 9.75; MS: $[\mathrm{M}]^{+}$at $m / z 431$.

5f $7^{\prime}$-Amino-2' $2^{\prime}$-dimethyl-2,4'-dioxo-5-methyl-1,2-dihydrospiro[indoline-3, $5^{\prime}$-pyrano $[2,3-d]\left[1^{\prime}, 3^{\prime}\right]$ dioxine]-6' ${ }^{\prime}$-carboxyethylester. IR $\left(\mathrm{cm}^{-1}\right): 3434\left(\mathrm{NH}_{2}\right), 3298(\mathrm{NH}), 3060$ (aromatic $\mathrm{C}-\mathrm{H}$ str), $1730(\mathrm{NH}-\mathrm{C}=\mathrm{O}), 1628(\mathrm{O}=\mathrm{C}-\mathrm{O}), 1188(\mathrm{C}-\mathrm{O}-\mathrm{C})$; ${ }^{1}$ HNMR $(\delta): 10.90$ (s, $1 \mathrm{H}, \mathrm{NH}$ indole), $8.12\left(\mathrm{~s}, 2 \mathrm{H}, \mathrm{NH}_{2}\right.$ ), 6.92-7.26 (m, 3H, Ar-H), 4.40 (q, 2H, $\left.\mathrm{CH}_{2}\right), 2.32$ (s, 3H, $\left.\mathrm{CH}_{3}\right), 1.32\left(\mathrm{t}, 3 \mathrm{H}, \mathrm{CH}_{3}\right), 1.12$ (s, $\left.3 \mathrm{H}, \mathrm{CH}_{3}\right), 1.10(\mathrm{~s}, 3 \mathrm{H}$, $\left.\mathrm{CH}_{3}\right) ;{ }^{13} \mathrm{CNMR}(\delta): 167.7(\mathrm{O}-\mathrm{C}-\mathrm{O}), 166.4(\mathrm{NH}-\mathrm{C}=\mathrm{O}), 166.1$ $(\mathrm{C}=\mathrm{O}), 159.2\left(\mathrm{C}-\mathrm{NH}_{2}\right), 124.3-142.0$ (aromatic carbons), 61.2 $\left(\mathrm{OCH}_{2}\right), 46.6$ (spiro carbon), $26.3\left(\mathrm{CH}_{3}\right), 24.1\left(\mathrm{CH}_{3}\right), 14.1$ $\left(\mathrm{CH}_{3}\right.$ ester); Anal. Calcd. for $\mathrm{C}_{20} \mathrm{H}_{20} \mathrm{~N}_{2} \mathrm{O}_{7}: \mathrm{C}, 60.00, \mathrm{H}, 5.03$, $\mathrm{N}, 7.00$. Found: $\mathrm{C}, 60.19, \mathrm{H}, 5.05, \mathrm{~N}, 6.99$; MS: $[\mathrm{M}]^{+}$at $\mathrm{m} / \mathrm{z}$ 400.

3.3. Characterization of the Synthesized $\mathrm{ZnO}$ Nanoparticles. The synthesized $\mathrm{ZnO}$ nanoparticles were characterized by using X-ray diffraction (XRD), FTIR, UV-VIS spectra, and fluorescence spectroscopy.

3.3.1. XRD Pattern of $Z n O$ Nanoparticles. The nanostructure of $\mathrm{ZnO}$ nanoparticle has been studied at room temperature by using X-ray diffraction pattern (Figure 2). The particle size was calculated from $\mathrm{X}$-ray diffraction images of $\mathrm{ZnO}$ powders using the Scherrer formula:

$$
D=\frac{K \lambda}{\beta \cos \theta},
$$

where $D$ is the average particle size perpendicular to the reflecting planes, $\lambda$ is the $\mathrm{X}$-ray wavelength, $\beta$ is the full width at half maximum (FWHM), and $\theta$ is the diffraction angle. The average size of $\mathrm{ZnO}$ nanoparticles obtained from the XRD is about $5.1 \mathrm{~nm}$, using the Scherrer formula.

3.3.2. Fourier Transforms Infrared Spectroscopy (FTIR). The FTIR was acquired in the range of $400-4000 \mathrm{~cm}^{-1}$ (Figure 3). The band between $450-550 \mathrm{~cm}^{-1}$ correlated to metal oxide bond $(\mathrm{ZnO})$. The peaks in the range $1400-1500 \mathrm{~cm}^{-1}$ correspond to $\mathrm{CO}$ bonds. The peaks at $1340 \mathrm{~cm}^{-1}$ and $1574 \mathrm{~cm}^{-1}$ correspond to $\mathrm{CO}$ and $\mathrm{OH}$ bending vibrations, respectively. 


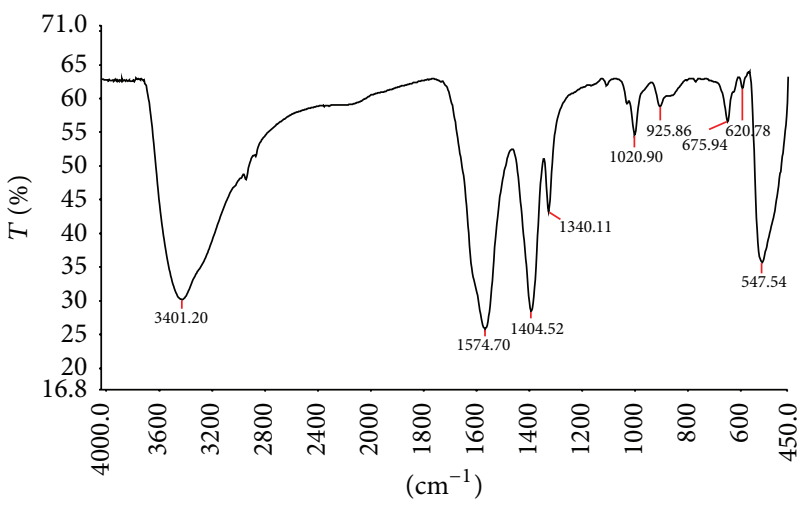

FIGURE 3: FTIR of $\mathrm{ZnO}$ nanoparticles.

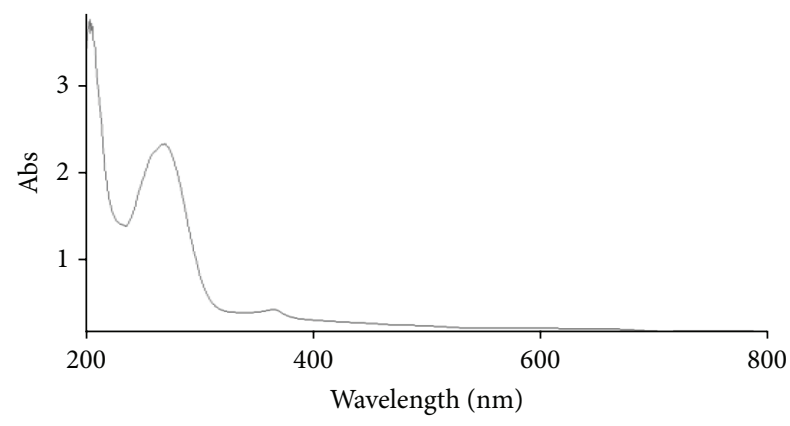

FIGURE 4: U-V spectrum of $\mathrm{ZnO}$ nanoparticles.

TABle 4: Fluorescence data $\mathrm{ZnO}$ in methanol at different molar concentrations.

\begin{tabular}{lcc}
\hline Entry & Molar concentration & $\begin{array}{c}\text { ZnO nanoparticle } \\
\lambda \text { em }(\mathrm{nm})\end{array}$ \\
\hline 1 & $1 \times 10^{-5}$ & 420 \\
2 & $2 \times 10^{-5}$ & 280 \\
3 & $3 \times 10^{-5}$ & 395 \\
4 & $4 \times 10^{-5}$ & 445,365 \\
5 & $5 \times 10^{-5}$ & 455 \\
6 & $6 \times 10^{-5}$ & 400 \\
\hline
\end{tabular}

IR spectra were recorded in $\mathrm{KBr}$ on a Perkin Elmer Infrared RXI FTIR spectrophotometer.

3.3.3. U-V Spectroscopy. The U-V spectrum was taken by using Cary 60 UV-VIS, Agilent Technologies. The sample was vigorously mixed through vortex for $10 \mathrm{~min}$. The U-V absorption spectrum of $\mathrm{ZnO}$ nanoparticle in methanol gave absorption peak at $275 \mathrm{~nm}$ (Figure 4).

3.3.4. Fluorescence Spectroscopy. The fluorescence spectrum of nano- $\mathrm{ZnO}$ at different molar concentrations in methanol was taken at different excitation wavelength $300-600 \mathrm{~nm}$ (Figure 5) (Table 4). All the samples were vigorously mixed through vortex for $10 \mathrm{~min}$. Fluorescence spectrum was recorded by using spectrofluorophotometer model number 5301PC, Shimadzu Cooperation, Kyoto, Japan.

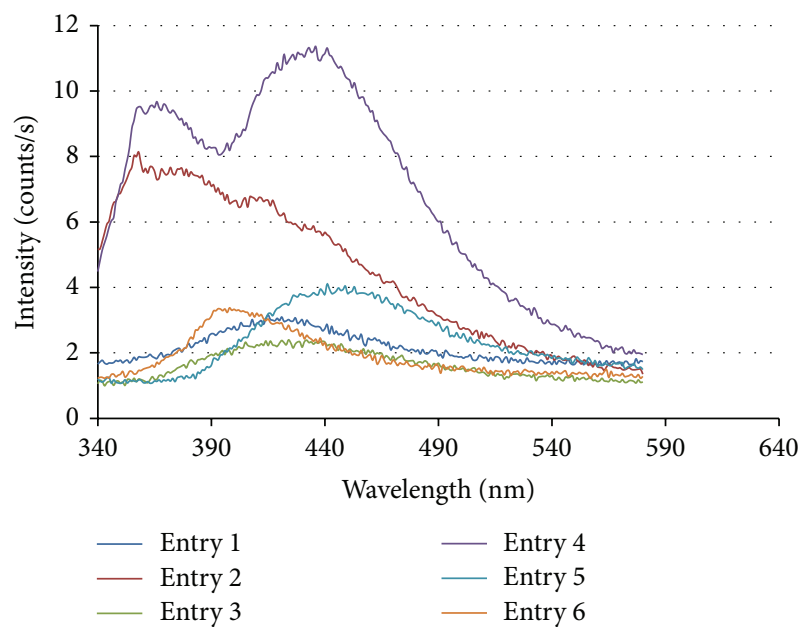

FIgURE 5: Fluorescence spectrum of $\mathrm{ZnO}$ nanoparticles.

\section{Conclusion}

We have demonstrated an environ-economic and simple protocol for the synthesis of novel spiroindole derivatives by the one-pot three-component reaction of isatin, malononitrile/ethylcyanoacetate, and Meldrum's acid with $\mathrm{ZnO}$ nanoparticles as a green, effective, and recoverable catalyst. The catalyst can be recycled and reused without apparent loss of activity.

\section{Conflict of Interests}

The authors declare that there is no conflict of interests regarding the publication of this paper.

\section{Acknowledgments}

The authors are thankful to the Dean and to the Head of the Department (Science and Humanities), FET, MITS, for providing necessary research facilities in the department. Financial assistance from FET, MITS, is gratefully acknowledged. They are also thankful to SAIF Punjab University, Chandigarh, for the spectral and elemental analyses.

\section{References}

[1] T. R. Bal, B. Anand, P. Yogeeswari, and D. Sriram, "Synthesis and evaluation of anti-HIV activity of isatin $\beta$-thiosemicarbazone derivatives," Bioorganic and Medicinal Chemistry Letters, vol. 15, no. 20, pp. 4451-4455, 2005.

[2] L. Chen, Y. Wang, W. L. Yi et al., "Synthesis and evaluation of isatin derivatives as effective SARS coronavirus 3CL protease inhibitors," Bioorganic and Medicinal Chemistry Letters, vol. 15, no. 12, pp. 3058-3062, 2005.

[3] P. Selvam, M. Chandramohan, C. E. De, C. Pannecouque, and M. Witrouw, "Synthesis and anti-HIV activity of 4-[(1,2dihydro-2-oxo-3H-indol-3-ylidene) amino]-N(4,6-dimethyl2-pyrimidinyl)-benzene sulfonamide and its derivatives," European Journal of Pharmaceutical Sciences, vol. 14, pp. 313-316, 2001. 
[4] N. Sin, B. L. Venables, K. D. Combrink, H. B. Gulgeze, K. L. Yu, and R. L. Civiello, "Respiratory syncytial virus fusion inhibitors. Part 7: structure-activity relationships associated with a series of isatin oximes that demonstrate antiviral activity in vivo," Bioorganic and Medicinal Chemistry Letters, vol. 19, no. 16, pp. 4857-4862, 2009.

[5] O. Guzel, N. Karali, and A. Salman, "Synthesis and antituberculosis activity of 5-methyl/trifluoromethoxy- $1 H$-indole-2,3dione 3-thiosemicarbazone derivatives," Bioorganic \& Medicinal Chemistry, vol. 16, pp. 8976-8987, 2008.

[6] N. Siddiqui, M. S. Alam, and W. Ahsan, "Synthesis, anticonvulsant and toxicity evaluation of 2-(1H-indol-3-yl) acetyl$N$-(substituted phenyl)hydrazine carbothioamides and their related heterocyclic derivatives," Acta Pharmaceutica, vol. 58, no. 4, pp. 445-454, 2008.

[7] I. Chiyanzu, C. Clarkson, P. J. Smith et al., "Design, synthesis and anti-plasmodial evaluation in vitro of new 4aminoquinoline isatin derivatives," Bioorganic \& Medicinal Chemistry, vol. 13, no. 9, pp. 3249-3261, 2006.

[8] S. Biswal, U. Sahoo, S. Sethy, H. K. S. Kumar, and M. Banerjee, "Indole: the molecule of diverse biological activities," Asian Journal of Pharmaceutical and Clinical Research, vol. 5, no. 1, pp. $1-6,2012$.

[9] U. C. Mashelkar and D. M. Rane, "Synthesis of some isatin based novel spiroheterocycles and their biological activity studies," Indian Journal of Chemistry B, vol. 44, no. 9, pp. 1937-1939, 2005.

[10] K. C. Joshi, R. Jain, and P. Chand, "Indoles with C-3 as spiro atom," Heterocycles, vol. 23, pp. 957-996, 1985.

[11] B. L. Palucki, S. D. Feighner, S. Pong et al., "Spiro(indoline-3,4' piperidine) growth hormone secretagogues as ghrelin mimetics," Bioorganic and Medicinal Chemistry Letters, vol. 11, no. 14, pp. 1955-1957, 2001.

[12] B. Rajashekhar and E. T. Kaiser, "Design of biologically active peptides with non-peptidic structural elements. Biological and physical properties of a synthetic analogue of $\beta$-endorphin with unnatural amino acids in the region 6-12," The Journal of Biological Chemistry, vol. 261, no. 29, pp. 13617-13623, 1986.

[13] D. H. Rich and E. T. O. Sun, "Synthesis of analogs of the carboxyl protease inhibitor pepstatin. Effect of structure on inhibition of pepsin and renin," Journal of Medicinal Chemistry, vol. 23, no. 1, pp. 27-33, 1980.

[14] S. Vassiliou, A. Mucha, P. Cuniasse et al., "Phosphinic pseudotripeptides as potent inhibitors of matrix metalloproteinases: a structure-activity study," Journal of Medicinal Chemistry, vol. 42, no. 14, pp. 2610-2620, 1999.

[15] S. J. Gould, "Streptonigrin," Progress in the Chemistry of Organic Natural Products, vol. 41, pp. 77-114, 1982.

[16] G. A. Kraus and P. K. Choudhury, "Synthesis of puraquinonic acid ethyl ester and deliquinone via a common intermediate," Journal of Organic Chemistry, vol. 67, no. 16, pp. 5857-5859, 2002.

[17] H. Y. Shi, B. Deng, S. L. Zhong, L. Wang, and A. W. Xu, "Synthesis of zinc oxide nanoparticles with strong, tunable and stable visible light emission by solid-state transformation of $\mathrm{Zn}$ (II)-organic coordination polymers," Journal of Materials Chemistry, vol. 21, pp. 12309-12315, 2011.

[18] J. Safaei-Ghomi, M. A. Ghasemzadeh, and S. Zahedi, "ZnO nanoparticles: a highly effective and readily recyclable catalyst for the one-pot synthesis of 1,8-dioxo-decahydroacridine and 1 , 8-dioxooctahydro-xanthene derivatives," Journal of the Mexican Chemical Society, vol. 57, no. 1, pp. 1-7, 2013.
[19] H. Alinezhad and S. Mohseni Tavakkoli, "Efficient and convenient synthesis of 1,8-dioxodecahydroacridine derivatives using $\mathrm{Cu}$-doped $\mathrm{ZnO}$ nanocrystalline powder as a catalyst under solvent-free conditions," The Scientific World Journal, vol. 2013, Article ID 575636, 9 pages, 2013.

[20] F. M. Moghaddam, H. Saeidian, Z. Mirjafary, and A. Sadeghi, "Rapid and efficient one-pot synthesis of 1,4-dihydropyridine and polyhydroquinoline derivatives through the hantzsch four component condensation by zinc oxide," Journal of the Iranian Chemical Society, vol. 6, no. 2, pp. 317-324, 2009.

[21] F. Tamaddon, M. A. Amrollahi, and L. Sharafat, "A green protocol for chemoselective O-acylation in the presence of zinc oxide as a heterogeneous, reusable and eco-friendly catalyst," Tetrahedron Letters, vol. 46, no. 45, pp. 7841-7844, 2005.

[22] B. V. Kumar, H. S. B. Naik, D. Girija, and B. V. Kumar, "ZnO nanoparticle as catalyst for efficient green one-pot synthesis of coumarins through Knoevenagel condensation," Journal of Chemical Sciences, vol. 123, no. 5, pp. 615-621, 2011.

[23] H. Sachdeva, D. Dwivedi, R. R. Bhattacharjee, S. Khaturia, and R. Saroj, "NiO nanoparticles: an efficient catalyst for the multicomponent one-pot synthesis of novel spiro and condensed indole derivatives," Journal of Chemistry, vol. 2013, Article ID 606259, 10 pages, 2013.

[24] K. Higashiyama and H. Otomasu, "Spiro heterocyclic compounds. III. Synthesis of spiro[oxindole-3, $4^{\prime}-\left(4^{\prime} \mathrm{H}-\right.$ pyran)]compounds," Chemical and Pharmaceutical Bulletin, vol. 28, no. 2, pp. 648-651, 1980.

[25] K. C. Joshi, R. Jain, K. Sharma, S. K. Bhattacharya, and R. K. Goel, "Studies in spiro-heterocycles. Part-XII. Synthesis of some fluorine containing spiro[3H-indole-3,4(4H)pyrano[2,3- $d$ ]pyrimidine]-2,5,7(1H)-triones as CNS agents," Journal of the Indian Chemical Society, vol. 115, pp. 202-204, 1988.

[26] Z. Sun, L. Liu, L. Zhang, and D. Jia, "Rapid synthesis of ZnO nano-rods by one-step, room-temperature, solid-state reaction and their gas-sensing properties," Nanotechnology, vol. 17, no. 9, pp. 2266-2270, 2006. 

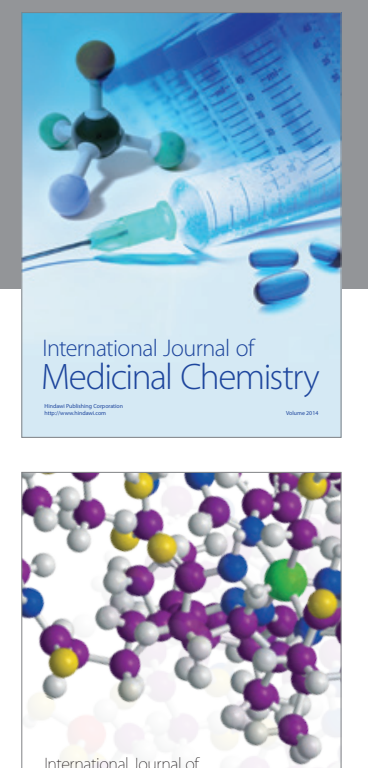

\section{Carbohydrate} Chemistry

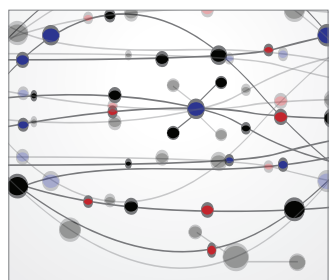

The Scientific World Journal
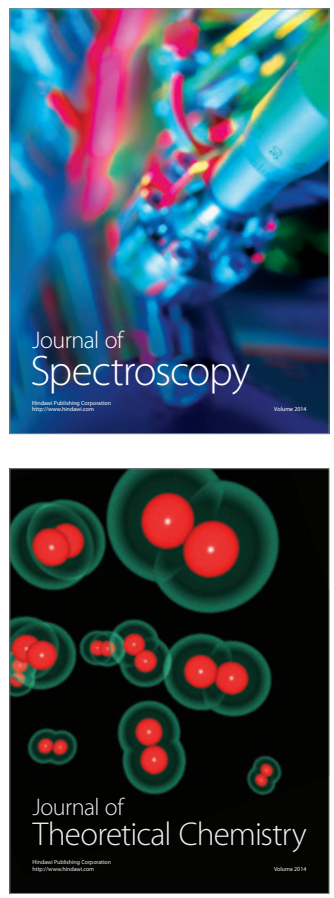
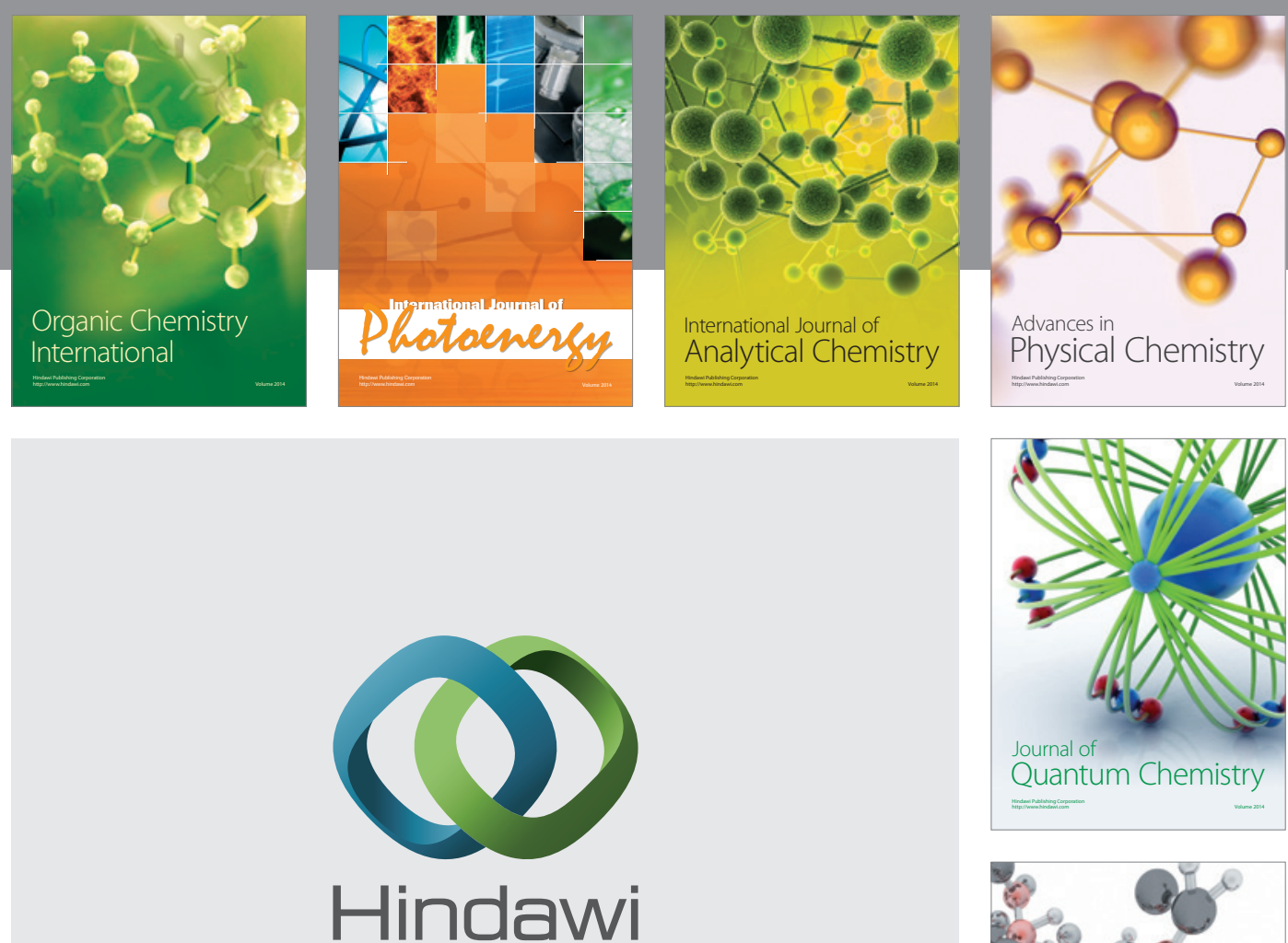

Submit your manuscripts at

http://www.hindawi.com

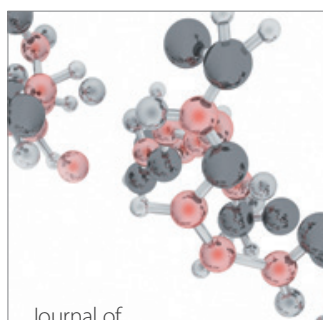

Analytical Methods

in Chemistry

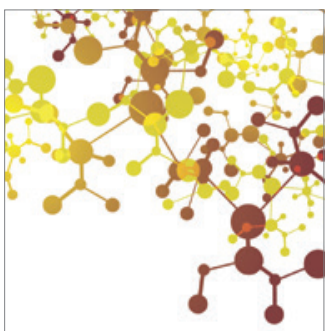

Journal of

Applied Chemistry

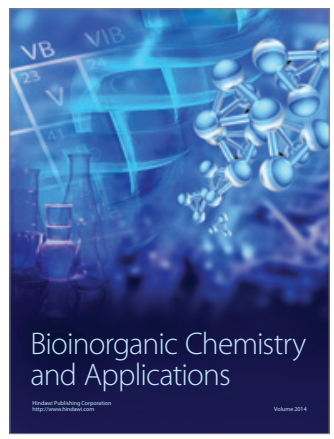

Inorganic Chemistry
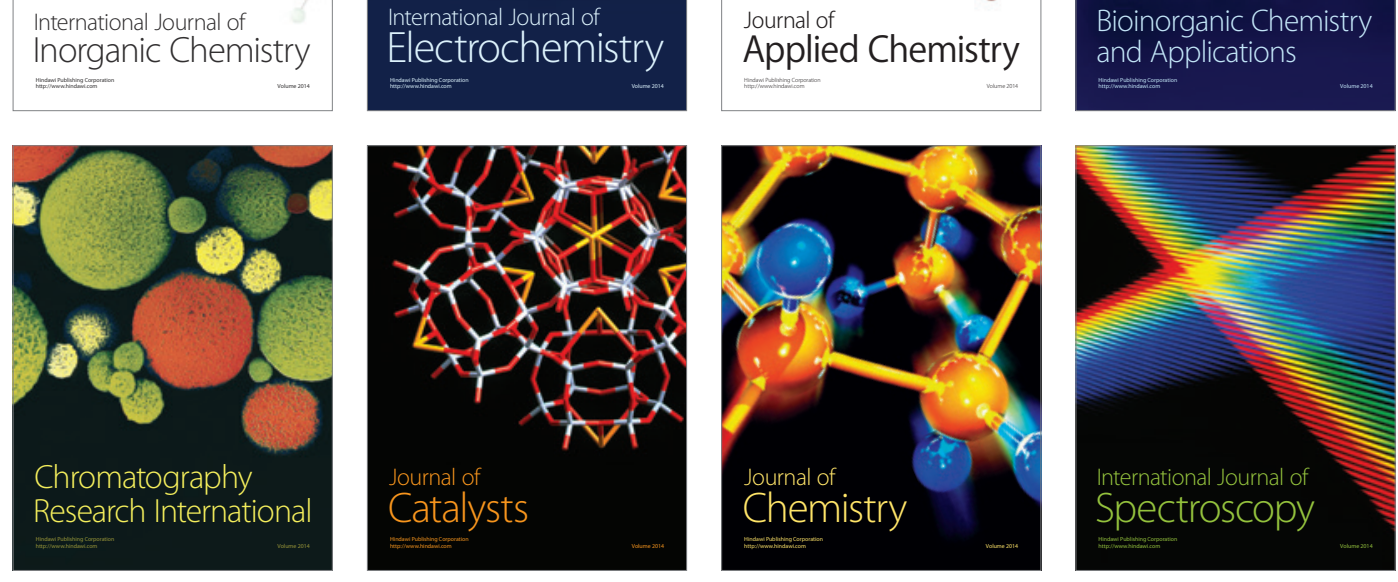